\title{
Choreographies of sperm donations: dilemmas of intimacy in lesbian couple donor conception
}

Petra Nordqvist, University of Manchester

Petra.Nordqvist@Manchester.ac.uk

Post peer-review version of article published in

Social Science and Medicine, 73(11): 1661-1668 2011. DOI:

10.1016/j.socscimed.2011.09.033

Please refer to the published version for citations.

This article draws on Petra's $\mathrm{PhD}$ research into lesbian couples' experiences of donor conception. For more information about other articles published by Petra about lesbian donor conception and assisted reproduction more broadly, please visit http://www.manchester.ac.uk/research/petra.nordqvist/

Petra's most recent project 'Relative Strangers' (2010-2013) (with Carol Smart) explored the impact of donor conception on family life among heterosexual and lesbian couples. The research explored parents as well as grandparents' experiences of donor conception in their families. The findings from this project are available in the book 'Relative Strangers: Family Life, Genes and Donor Conception'. If you are interested in reading more about the project and see our videos, please visit http://www.socialsciences.manchester.ac.uk/morgancentre/our-research/kinship-andrelatedness/relative-strangers/ 


\title{
Choreographies of sperm donations: dilemmas of intimacy in lesbian couple donor conception
}

\begin{abstract}
Assisted conception involving donor insemination challenges cultural idioms of parenthood and family; there is now a growing body of work exploring how women and couples negotiate becoming a family in this way. But sperm donation also raises questions on the more intimate levels of sex, sexuality and sexual bodies, and these have received little sustained attention in the literature. Lesbian couples in the UK increasingly negotiate access to medicalised donor insemination, but many also conceive in informal arrangements with donors where they themselves negotiate proximity and contact with donors when retrieving donor sperm. I explore in this paper how lesbian couples manage and perceive sperm donations, seek to negotiate their intimate, sexual and bodily overtones, and how the medical and non-medical settings enable them to do this in different ways. I draw on empirical data from an interview study conducted from 2006-2009 in England and Wales comprising 25 lesbian couples. I suggest that sperm donation raises dilemmas of intimacy for lesbian couples, and that couples try to resolve such dilemmas by carefully and intentionally choreographing donation events through managing patterns of movement and action. The different institutional, medical and regulatory frameworks governing clinical and non-clinical sperm donation shape that management in significant and different ways. I argue that sperm donation choreographies enable couples to negotiate the private, sexual and intimate tensions surrounding sperm donations, and also the subjectivity of the sperm donor.
\end{abstract}




\section{Introduction}

Increasingly, men and women are utilising reproductive technologies and also donated eggs, sperm or embryos to conceive a child. Assisted donor conception challenges the cultural idioms of parenthood and family, and there is now a growing body of work exploring how donor conception alters the social and cultural discourses of family, kinship, connectedness, as well as the sociological and psychological dimensions embedded in becoming and being a family in this way (e.g. Becker 2000, Daniels \& Haimes 1998, Donovan and Wilson 2008, Edwards 2000, Murray \& Golombok 2003, Hargreaves 2006, Strathern 1992). Donor conception, however, also raises questions of a more intimate kind to do with bodies and bodily fluids, sexual practices, and interpersonal relationships. These dimensions of donor conception have to date received much less attention in the literature.

Sperm donation, in particular, gives rise to dilemmas of intimacy. It transports the intimate, personal and private act of producing sperm from the private sphere into the public domain. Notwithstanding that sperm production in the context of a donation may be defined as non-sexual, non-intimate and undertaken with the explicit purpose of producing semen specimens for (somebody else's) reproduction, it is also inextricably linked to male masturbation, ejaculation and sexual practice. As such it evokes, culturally and socially, sexual images (Kirkman 2004). Sperm donation is a social activity that risk evoking contradictory and competing definitions of reality (compare Emerson 1970) and recipients and donors may understand and define the event in different ways. This paper explores how recipients may negotiate these processes.

Sperm donation takes place in the context of reproductive health centres, but it is also undertaken privately in so-called self-arranged conception. The latter is particularly common among lesbians, who have been known to conceive in this way since the 1970s (Luce 2010). This is likely to be linked in part to the legal restrictions that have, until recently, denied lesbian access to UK infertility clinics (e.g Barney 2005). In recent years, lesbians increasingly access donor conception through reproductive health centres. The Human Fertilisation and Embryology Authority, which licences reproductive health clinics to perform insemination using a donor in the UK, requires clinics to freeze donated sperm for at least 6 months in order to screen the sperm and the donor medically. This regulatory framework also sets out 
that donors are anonymous to women or couples who use assisted conception and they are not considered the legal father of any child produced from their donation. While lesbian couples in the UK increasingly negotiate access to reproductive health clinics, many also conceive in informal arrangements with donors where they themselves have to negotiate receiving sperm donations. Such self-arranged conception practices are privately organised donation arrangements where sperm is 'freshly' donated and inseminated. Sperm only survives for a limited time outside the body in open air (around 1 hour 20 minutes), and so the semen sample must be quickly handed over after ejaculation, meaning that lesbians themselves have to negotiate close contact with donors as part of that process.

Drawing on an empirical study of lesbian couples' pursuit of donor insemination inside and outside reproductive health clinics, I explore in this paper how recipients of donor sperm perceive and negotiate the sexual and intimate dimensions inherent to retrieving sperm donations, and also how the non-clinical and clinical context shapes these negotiations in different ways. I suggest that sperm donations are carefully structured and organised social practices which are meticulously choreographed through patterns of movement and action. I argue that these choreographies are rituals that represent complex negotiations of intimacy, privacy and sexuality but also subjectivity, interpersonal relationships and personhood, and which are aimed at stopping sperm donations spilling over sexual and intimate boundaries. I further argue that the non-clinical and the clinical context shape these choreographies and rituals in specific and significant ways.

This paper discusses how recipients manage the sexual and intimate overtones of retrieving donor sperm. Its task is to explore how couples manage bodily and sexual proximity with donors, and how this may vary with context. Although this also links in with how couples perceive and negotiate their own intimacy as a couple, this will for reasons of space not be discussed here, but will be the focus of a forthcoming paper. It is also not the task of this paper to discuss how couples' negotiations of private and sexual dimensions relates to their perception of the donors' potential parental identity and genetic contribution, or their family building practices at large (this has been discussed elsewhere, e.g., Donovan and Wilson 2008, Ryan-Flood 2009). It is worth noting, however, that although the couples sought to distance themselves from the sexual dimensions of sperm production in the process of 
retrieving sperm, this did not necessarily translate into them rendering the donor invisible in a wider sense (Nordqvist under review).

\section{Theoretical framing}

There is now a body of work exploring the psychological, sociological and anthropological dimensions of assisted donor conception, and this is increasingly widespread and diverse. This paper is framed, in particular, by four areas of debate. These are the medicalisation of infertility; negotiations of subjectivity, personhood and objectification in assisted reproduction; lesbian donor conception practices; and the social anxieties associated with sperm donations.

Medicalisation represents one of the most significant transformations of the $20^{\text {th }}$ century, and is intimately linked with and co-constitutive of modernity and the enlightenment (Clarke 1995, Clarke et al 2003: 16, see also Latour 1993). Following Clarke et al (2003) I understand it here as the process through which aspects of life, which have previously been seen as outside the framework of medicine, become construed as medical problems. Medicalisation has been highly influential in the field of conception, reproduction and involuntary childlessness (Mamo 2007). Mamo highlights that for most of human history sterility was viewed not as a medical, but as a social and moral issue, linked to self-induced immorality (such as abundant sensuality, idleness or depression) (Pfeffer 1993 in Mamo 2007). With processes of medicalisation, and since the end of the $19^{\text {th }}$ century, sterility has been recast as a biological pathology, rendering unwanted childlessness a medical problem. The medicalisation of involuntary childlessness has given rise to a number of medical developments aimed at understanding and controlling reproductive processes and biomaterials, including sperm. A number of techniques now enable sperm to be stored, analysed, washed and also cryopreserved (frozen and thawed) (Mamo 2007). Laboratories for screening, preparing, storing and distributing sperm are now commonplace, and from the late 1970s onwards assisted conception (as it is now known) has grown as a medical area of interest and is now a sizeable industry. With infertility medicine expanding in the late $20^{\text {th }}$ century, feminist scholars became increasingly critical of the medicalisation of reproduction. They saw it as patriarchal violence that resulted in the control, exploitation and objectification of women (for an overview see Michelle 2006). This analysis of objectification has, however, been challenged in more recent work that explores women's own 
experience of the technologies, notably Thompson (2005) (but see also Franklin 1997). Thompson (2005) argues for a complex understanding of the relationship between objectification and subjectivity in assisted reproduction. She suggests that medical technology can stand both in partnership with and in opposition to women's subjectivity and agency. She argues that technology enhances subjectivity when body parts, which are objectified through the medical gaze in treatment, come to 'stand in' for the woman in a synecdochal relation. The technology is perceived to enhance her subjectivity because it contributes to her overall goal of achieving pregnancy. For this to be successful, the objectified body parts must not lose their metonymic relation to the whole woman and her personal trajectory of pregnancy. Thompson argues that connections, such as that between the treated woman and her body parts, are maintained or construed in this context through a so called 'ontological choreography'; a highly organised and carefully balanced coming together of aspects of different ontological orders (2005: 8). Objectification, on the other hand, occurs when the connection between the person, the body parts and the personal trajectory is lost. Technology can thus be understood to relate to subjectivity, personhood, agency and objectification in complex and context-specific ways.

Lesbians have been affected in specific ways by the medicalisation of involuntary childlessness. With a history of self-arranging conception, in part linked to being excluded from medical reproductive health centres, lesbians now increasingly access medicalised treatment (Luce 2010, Mamo 2007, Nordqvist 2011). Michelle (2006) notes that the medicalisation of infertility, defined as biological pathology, has resulted in uneven provisions of reproductive health care to lesbians. Luce (2010) demonstrates that lesbians' reproductive processes have been both enabled and constrained by changing medical, social and legal discourses. She, and also Mamo (2007), suggest that the boundaries between non-medical and medical practices, and high-tech and low-tech conception, are fluid in lesbians' conception practices.

Notwithstanding that donor insemination has become part of the jurisdiction of medicine, socially and culturally it remains a fraught and anxious practice. Kirkman (2005) notes that donor insemination has historically been rendered invisible and veiled in secrecy. She indicates that the combination of sin and embarrassment associated with sperm donors, and resorting to donor insemination in order to conceive, are powerful disincentives to openness. Kirkman (2004) further shows that heterosexual recipients of donor sperm perceive the donor in ambivalent ways. They 
feel gratitude towards him but also embarrassment; the donor is seen as a kind man as well as a wanker. Grace, Daniels and Gillett (2008) suggest that parents seek to distance themselves from the donor, denying his existence as a person. Their work indicates that a distancing effect is reinforced through the medicalised processes of screening, testing and sanitising sperm. These findings are echoed in Burr (2009) who notes that although donors are anonymous, they linger in the imagination of the participants. Recipients, therefore, can feel ambivalent about retrieving donor sperm at the time of conception, but also after birth and as their children are growing up. Mary Douglas' (1966) work on dirt and disorder has been utilised in some of this research to explain the anxieties associated with sperm donation (see Burr 2009, Donovan 2006).

\section{Data and methodology}

The study comprised 25 in-depth semi-structured interviews with a total of 45 women in England and Wales with experience of pursuing parenthood using donor sperm as a lesbian couple. Previous studies of non-heterosexual life note that same-sex couples constitute a 'hidden' population and a hard-to-reach group (Weeks et al. 2001), meaning that random sampling is not an option. A purposive sample based on selfselection was employed in this study, and couples were primarily recruited using online gateways. These included, e.g. 'Rainbownetwork' (www.rainbownetwork.com) and 'Gingerbeer' (www.gingerbeer.co.uk). Both the sample size and the method of recruitment meant that couples in this study were unlikely to be a representative cross-section of the UK population of lesbian couples. Themes that emerged from the interviews, however, were likely to have generalisability beyond the sample from which they were drawn, as the data offered in-depth understandings suggestive of the ways in which these processes are also experienced by others (Mason 1996).

Fieldwork took place from September 2007 to March 2008 and a qualitative approach was utilised. The interviews were theme-based and guided by questions about the experience and understanding of planning conception, undertaking the insemination, the couple's relationship, and becoming and being a family. All interviews were recorded and transcribed verbatim and the data were thematically analysed using a narrative-holistic approach (Lieblich et al. 1998), conducted through the graphic analysis of event-state networks (Miles \& Huberman 1994). This entailed 
the construction of graphic 'maps' detailing the couples routes to conception by marking the special events (e.g. met donor, visited clinic) and states (e.g. feelings, desires, convictions) that lead up to these events. From the networks, codes and analytical themes were derived. The study raised ethical issues of anonymisation, confidentiality and researcher safety and was approved by the Centre for Women's Studies Ethics Committee, University of York. Names, places and identifying details in the interview extracts have been altered to protect participants' anonymity.

Twelve of the 25 couples tried to conceive or had conceived using clinical conception, while eleven engaged in self-arranged conception and a further two were planning future conception. Nine couples had at some point tried both routes. Fourteen couples were parents of donor conceived children, the children being between three months and 7 years old (an additional four couples were pregnant with their first child). In four cases women were also parents of children conceived or adopted in previous heterosexual relationships.

The women were between 23 and 56 years of age (median age 33.5 years). Fortytwo women identified as white British, Welsh or English and three women identified as of either mixed ethnic origin, Chinese British or Black British. Following Graham (2007), education was used as a measure of class linking parental social class and the respondents' own class. Thirty-six percent of respondents had left school at 18, while 64 percent had a higher education qualification, suggesting that more than one third of respondents had working-class backgrounds while the others were from middle-class backgrounds.

\section{Choreographing sperm donations}

\section{Negotiating self-arranging sperm donations}

The couples who self-arranged their donor conception planned the donation and insemination processes independently of a reproductive health centre. The women mapped the birth mothers' menstrual cycle and each time she ovulated they arranged to meet up with the donor. Recipients and donors often lived far apart, which meant undertaking extensive travelling and occasionally overnight stays. In most cases, it took many attempts to conceive, and so the process of meeting the donor and receiving a donation was often repeated over many months and sometimes years before successful conception. During this time, the couples tried to maintain working relationships with donors; it was evident that finding a suitable donor was so difficult 
that once an agreement had been established, the couples were reluctant to revisit the arrangement. A small number of couples in this group had friends who acted as donors, but the majority used donors whom they found online. These were men who they did not know beforehand, and whom they wished would remain a distant and detached figure.

The desire for distance was in direct tension with the practical process of retrieving sperm, and the need to inseminate the semen quickly while it was mobile. As a result, recipients' and donor's personal and also intimate boundaries would easily blur and mesh. Anna and Sally's narrative usefully introduces the dilemmas experienced by couples. I explore their account at some length because it highlights the complex issues involved in managing sperm donor arrangements while maintaining interpersonal boundaries.

Anna and Sally could not afford the costs of a clinic, and found a donor online who they wished would remain as detached as possible. Speaking about how they saw the donor in relation to their conception and family life, they said:

Anna We don't want him feeling like...

Sally ... [he is] part of the family. Because [donors] are not sort of thing you know. And I know that sounds really cruel but...

Anna I don't want that.

Sally If we keep up a rigid barrier, then they will always know that that barrier is there. And there is no starting let in (Anna, 32 and Sally, 33).

Anna and Sally lived in the southwest of England and their current donor lived in the north (a distance of about 300 miles). At the time of each donation, the donor would come and visit the couple for a few days. Anna and Sally had used other donors before their current one, whom they had met at Anna's mother's house.

Anna We have invited them [to mum's house] and it was sort of like, do you want a coffee or something. And we usually have a little chat for about half an hour to an hour. On the first meeting, about half an hour. But with [this donor] now it is about two hours. But that is only because he drives for four hours. And I can't just go right, get in there, get out. You know what I mean.

Sally I did make him a sandwich yesterday and a cup of coffee. 
As the interview progressed it became increasingly clear that, with this donor, the desired 'rigid' barrier became difficult to uphold in practice. Over time, and with repeated donations, Anna and Sally got to know the man well, and developed a friendly personal relationship with him. Maybe this is why Anna and Sally told me with some emphasis that notwithstanding now being friends, they tried to maintain personal and intimate distance. The donor would travel to where the couple lived, where he would stay as a guest in one of the women's two apartments. Anna indicated that she organised the sleeping arrangements in carefully planned ways:

Although he slept at our other place down the road, I got the sofa bed out for him. He wouldn't sleep in our bed. I got the sofa bed out in a different room. We weren't in the same house as him and he wasn't in our bed. So it was like he, almost staying in a guesthouse really (Anna).

It is clear from Anna's account that she perceived this to be important, and that it would be inappropriate if certain boundaries were crossed. Morgan (1996) suggests that family and intimacy are constructed through every day practices, including the regulation and negotiation of domestic space and temporality. Such space is both shaped by and shapes personal boundaries and intimate relationships. Anna's management of space can be understood as a way of creating intimate barriers towards the donor, signalling that he was not part of their family.

Anna and Sally's narrative indicates that they engaged in a careful management aimed at maintaining personal and intimate boundaries. The process of retrieving sperm emerges as somewhat risky and precarious; a process that may go wrong if not carefully planned. Their definition of reality and the 'correct' version of relationships are defined and defended. This is done through the creation of symbolic barriers of separation and delineation: Anna made distinctions between areas in the house, different furniture and being home/away. Mary Douglas (1966) suggests that processes of separation carry important cultural meaning in that they create order when things appear ambiguous, unclear and blurry:

Separation and demarcation have the main function to impose system on an inherently untidy experience. It is only by exaggerating the difference between within and without, above and below, male and female, with and against, that a semblance of order is created (Douglas 1966: 4). 
Following Douglas, Anna and Sally's management of space can be understood as symbolically loaded patterns or 'rituals' that gain meaning socially because they 'cancel' the risk perceived to lie inherent in the retrieval of donor sperm. It appears that sperm donation in a non-clinical context is perceived as somewhat messy, unclear and ambiguous, and thus couples are required to think carefully about how to undertake the process. Mary Douglas (1966) suggests that notions of mess and disorder culturally link in with the transgression of socially valued boundaries. Rather than understanding disorder as a status that resides in an object or process itself, she suggests that things become perceived as disorderly because they transgress social and cultural boundaries. Disorder - or 'dirt' - is seen as risky and dangerous.

Drawing on Douglas' framework, my data indicates that these couples perceived retrieving a sperm donation in a private context, outside the medical framework, as an anxiety-provoking and risky practice. The recipients organised sperm donations as carefully choreographed events. The donor's and their own movements in space and time were meticulously managed and planned. Mistakes were experienced as morally and emotionally loaded. Holly and Carol used a friend as donor when trying to conceive and indicated that it was far from arbitrary where the donation would take place:

Holly We said he couldn't go into the spare room because that had got all [my niece's] things in there, and like little... she's got a little bed and everything in there and that felt...

Carol I don't know why that would seem so wrong but it did.

Holly That felt more wrong than him going into our room. And he was the one that said, no, no, I'll go in the bathroom, because that's a neutral space. That's what he said. [...] But I was happier when he said he'd go in the bathroom. Just in case he spilt something on the bed really (Holly, 28 and Carol, 32).

Another couple, Rachel and Amy, had tried to access a reproductive health centre but were refused treatment as lesbians. They had found a donor online, and wanted a detached relationship. At the time of the first donation, the donor invited them into his home, and suggested that they could watch TV while he masturbated in another room. The couple reported having felt extremely uneasy about doing so, but agreed because 
they felt unable to refuse without risking the donation. However, they tried to manage the next cycle differently:

Amy At the time when [our daughter] was conceived we tried to avoid this thing so I text him or rung him as we were coming in to [City] said we will be about 15 minutes off you go. So then we arrived and he lived on like a top floor flat. So I tooted out, rung the bell. And he came to the intercom thing and said oh I haven't finished. It was like oh God. So then he says do you want to come in? I had to go all the way up to this top floor flat and sit, on the stairs outside his flipping door waiting for him to finish doing... Oh it was awful.

Rachel The first two were much better because they brought it, pre-done and that was it. But with him he got a bit of a kick out of actually knowing that we were there waiting (Amy, 28 and Rachel, 33).

The attempt to control movements in time and space, between different domestic rooms, upstairs and downstairs, inside and outside, and in time, brings to mind a carefully executed and structured dance, that aims to contain and control various aspects of the donor, and the donation. Holly and Carol's account about donating in the toilet evokes sentiments of sperm production in this context as contagious; donor sperm must not be produced, spilt or be seen to exist in certain domestic spaces (loaded with particular relational meaning). Douglas (1966) notes that cultural understandings of 'dirt' are particularly associated with the margins of the body, such a menstrual blood or semen. The danger of sperm is so strong that it is not called by name (Holly calls it 'something') although the exchange of the bodily fluid of sperm is the sole purpose of the interaction. A notion of sperm as dangerous and pollutive was typical in the accounts, but emerged with particular clarity in my interview with Holly who noted that the donor insemination also influenced her and Carol's sexual intimacy:

Once Carol had had the sperm I didn't touch her for probably a week after. I just... the thought of getting sperm on me, it's just like not a nice thought really for me. So I'd stay away because it's just better. 
The sperm, and its association with the male body (Martin 1991), was experienced as biological matter 'out of place' in the context of Carol and Holly's relationship, and required careful management if its pollutive power was to be contained.

In addition to managing the pollutive power of donor sperm, the choreographies were also aimed at maintaining distance between the couple, the donor, and his sexual practice. Amy and Rachel's account about keeping a temporal distance (above) alludes to the fact that waiting for a donation attached them in very concrete ways to the act of producing the sperm. Temporal (and spatial) distance, however, could to some extent 'hide' the production of sperm, the donor's sexual practice and his personal desires. The couples in my study constructed donations as non-sexual by managing details of the scene in which it was undertaken (Emerson 1970). Ambiguity was not tolerated, so the way in which the process was managed in time and space could require adjustment if boundaries were to be maintained.

Although the couples in my study sought to choreograph the sperm production and donation process, things did not always work out as planned. Such cases, in particular, highlight just how managed these processes were, and how important it was for the couple to get the rituals of the event 'right'. Joanne and Pippa had thought carefully about the process. Pippa noted in the interview that they wanted a detached donor. In their minds, the donation was strictly impersonal and non-intimate: the couple wanted a baby and the donor's part in that was reduced to producing a pot of sperm. They had agreed with the donor that he would come to their home, do the sperm donation in the bathroom and then go for a walk as the couple inseminated in the bedroom. However, just before the donor's first visit he phoned up, and Pippa recalls this phone conversation:

He said well maybe I can do it in the bedroom. And I went, right [hesitates]. Ok you know. I just thought oh, you know in our bedroom. [...] And then he said, I don't know, I think it is quite impersonal what I'm doing. And we said yeah that is the nature of what you are doing. And he said well, I wonder whether you could both be there. And [...] I went sorry what? And he said oh I wonder whether you'd be there. You know whether you'd sit on the bed while I'm doing what I'm doing so it would feel a little more personal to me. And I just... and I jokingly said what, you know, what are you talking about? And he said well I wouldn't get you to touch me or anything. I just want you in the room. And I was like right ok. You know and I wouldn't want you to do anything you 
know maybe you could take your top off (Pippa, 35, trying to conceive together with Joanne, 26).

Pippa's narration of this event signals how she distances herself from every suggestion about personal, intimate and sexual closeness made by the donor. As the couples' choreography of the event broke down, so did the semblance of order and the carefully constructed separation and delineation between the couple and the donor, and between them and his sexual practice and personal desire. With the donor and his sexual body being visibly attached to the sperm, and his agency, subjectivity and sexual desire moving to the forefront of the process, the event, for the couple, collapsed into a pollutive, disorganised mess. As a result, it became untenable. Despite having invested more than 6 months of work in building up a relationship with this donor, Joanne and Pippa broke off the arrangement shortly after receiving this phone-call. Other couples also reported feeling extreme unease and discomfort if the planned choreography broke down or was challenged by donors, for example if the private nature of the arrangement could not be maintained and the stages of the donation process came fully into public view. Douglas (1966) suggests that separation strategies aim to uphold structural social distinctions. What collapsed when these choreographies fell apart were the boundaries between private and public, between heterosexual and homosexual and between monogamy and polygamy. I argue that the extreme discomfort and unease reported by Joanne and Pippa resulted from the fact that the sexual, intimate and personal dimensions intrinsic to sperm donations became fully enmeshed and inseparable from the sperm itself, and the couple receiving it. Consequently, the links between the couple's conception and the donor's masturbation became startlingly obvious with the result that the couple could no longer manage to maintain a distant relationship with the donor.

I argue that for these processes to be experienced as successful by the couples, they had to be executed in ways that emphasised the separation and delineation between the donor's masturbation and the lesbian couple, hid the donor's personal desires, and also contained the pollutive power of donor sperm. I argue that this separation was important because it made the connection between the production of sperm and the couple tenuous and as minimal as possible; it enabled the recipients to perceive the two as disconnected and unrelated. This perceived disconnection was achieved as the production and the retrieval of sperm was drawn out over time and space, creating a 
delay between the donor's masturbation and ejaculation, and the sperm that was handed over. I argue that this delay was essential to establish the sought after boundaries and a semblance of order. Building on this insight that drawing out, delineating and delaying carried intimate meaning for these couples, I shall now discuss how similar processes, patterns of behaviour and meaning around sperm donations were produced in the context of reproductive health centres.

\section{Retrieving sperm in the reproductive health centre}

The legal framework governing clinical sperm donation in the UK until April 2009 was the UK Human Fertilisation and Embryology Act 1990 (this was revised in 2008). The regulatory framework from 1990 stipulated that donors remain anonymous to couples who accessed licensed donor sperm, a framework that linked in with donor insemination and donors being historically rendered invisible (Kirkman 2005). Donors were by law not seen as the natural father of the child, and children conceived prior to 1 April 2005 were unable to seek any information about their donors. These regulations meant that donors were entirely anonymous to the recipients during the conception process. Additionally, they mediated various practical arrangements at the clinic. It was medical staff (rather than the couples) who recruited donors and communicated with them. Donors and couples never met and had no personal knowledge of each other. Prior to purchasing vials of sperm, the couples' knowledge of the donor was limited to a select few physical characteristics.

The retrieval of sperm in clinics was also highly medicalised and the sperm went through processes of screening and analysis as well as washing, storing, freezing and thawing (compare Mamo 2007). These processes were all undertaken by medical staff. This meant that couples never had to directly negotiate the routines for the production of donor sperm or physically handle the sperm.

In the reproductive health centre, the regulatory framework and staff set out the donation procedure. The HFEA guidelines for the 'men's room' in UK clinics state that '[a] centre should ensure that its clinical facilities provide privacy and comfort for those [...] producing semen specimens' (Human Fertilisation and Embryology Authority Code of Practice $8^{\text {th }}$ Edition, section 25:11). A publicly available inspection document from a 2006 license renewal report highlights that the 'men's room' was judged on its suitability and the inspector had found 'the men's room to be "private but not ideal"', recommending it as an 'area of improvement' (Human Fertilisation 
and Embryology Authority License Renewal Inspection Report for Treatment and Storage Centres, 2006: 13). The terminology of 'men's room' highlights how masturbation and sperm donations are managed through metaphoric language and are hidden from view in carefully managed private spaces.

Thompson's (2005) ethnographic study of infertility clinics (in the U.S) enables us to get a sense of how clinical staff negotiate sexuality and privacy in practice.

Although Thompson writes about heterosexual couples where the male partner's sperm is collected for testing, her account is suggestive of the conscious choreography of the events:

At one typical clinic, the examination room furthest away from the nurses' station was assigned for male patients to collect [sperm]. It is out of the line of sight of any of the offices. [...] For collection, the 'in-use' light is put on, and no one can break the seal of privacy except the patient himself, which he does by exiting. He is instructed to lock the door from the inside to avoid error. [...] When [the technicians] hear the door open [by the man exiting], they move to the door of the room to make sure that the man can hand his container straight to somebody who will deal with it technically. The transmission from private and sexual to an appropriate clinical object is thus smoothly ensured (Thompson 2005: 110f.).

Following Thompson, the spatial and temporal choreographies in the clinic enabled the sperm to transform from being sexual and private to becoming a safer, less ambiguous, clinical object.

Both of these examples illustrate how privacy is carefully managed in the context of the infertility clinic in ways that are not dissimilar to lesbian couples' choreographed self-arranged conception. However, the institutionalised character of the clinic setting, and its legal and medical frameworks, meant that the clinic and the state, rather than the couple, handled issues to do with safeguarding privacy and the dangers associated with sperm. The production of sperm was significantly further removed from the couple through the anonymity framework, but also through the introduction of a number of medical procedures of analysing, washing, freezing and thawing sperm. Together, these processes produced a set of intermediary procedures that amounted to a process of separation. Consequently, this produced a drawing out effect, disconnecting the sperm from its production and from the donor himself. 
My interviews with lesbian couples suggest that this drawing out process produced important effects for the couples, and that it made retrieving sperm less fraught with tension and danger. They had not communicated with the donor and were unaware of who he was, his personality, or his personal desires. They had not been present when he had masturbated, were unaware of when and where he had done so, and had not handled or touched the sperm. Instead, what they experienced was a highly medicalised process where the medical staff and clinical medical practices acted as intermediaries between the couple and the production of sperm. Linda's account indicated that this framework impacted on how she perceived the donation:

It was quite impersonal at the hospital which is good whereas it all coming in fresh [in non-clinical conception], it's something... it makes it all a bit more squeamish, if you know what I mean, when you have to deal with it yourself. [...] I don't know, there's something about it being done in a hospital that sort of takes all of that nastiness... I think, being a lesbian, it's the last thing you really want to be involved in, isn't it? [...] You don't want to be dealing with sperm at all. Let somebody else deal with it.

The choreography integral to the reproductive health centre made the sperm donation more impersonal and more "thing-like" in the eyes of this lesbian couple. Many preferred this and saw self-arranged conception as more 'risky'. Gillian, for example, said that she preferred clinical conception because self-arranged conception was a bit 'seedy' because it was 'almost like using a penis'. The couples produced and perceived lines of difference between clinical and non-clinical conception based on the ability to distance themselves from the production of donor sperm and the male sexual body. Grace, Daniels and Gillett (2008) suggest that the medical processes of screening, testing and sanitising sperm enable couples to distance themselves from the donor as a person, making him invisible. In similar ways, the lesbian couples were able to distance themselves from the donor and the sexual, intimate and private dimensions of sperm production through the medical procedures. These findings add in important ways to the findings of Burr (2009), Grace, Daniels and Gillett (2008) and Kirkman (2004) in highlighting that the management of donor insemination not only carries important relational effects in terms of rendering the donor invisible, but that it also carries important meanings for the management of intimacy, bodies and bodily fluids. 
I argue that the rituals embedded in the medical context, through which the production of sperm was separated and distanced from the insemination, amounted to a process of purification where the product sperm was detached from its source of origin and rendered less threatening. Latour (1993) argues that processes of purification are fundamental to a notion of modernity and enlightenment, including the institution of medicine. He suggests that modern society has constructed an imagined separation between nature and culture. This has, according to Latour, never been achieved. What has been achieved is a perception of separation. Following Latour, this perception is produced through processes of 'translation' that create hybrids between nature and culture and second, practices of 'purification' that institute distinctions between the two, rendering the hybrids invisible. Practices such as assisted reproduction are inherently hybrid practices that are intrinsically both human and non-human; part of both nature and culture. Building on Latour's (1993) work, I argue that donor sperm has, through translation processes, required a hybrid status as social, personal, private intimate, sexual, human and biological. However, within the medical framework it undergoes a purification process through which it is separated from culture and becomes a medical and biological object; it is stripped of its personal dimensions and rendered a 'pure' biomaterial object. The production of sperm, and its linkages with intimate, sexual, personal and subjective trajectories, is 'black-boxed' (Thompson 2005: 47). This 'black-boxing' is achieved through a number of interlinked practices related to the law of anonymity, recruitment procedures, a raft of medical procedures, and the activities of medical staff. As a consequence, lesbian couples are able to disconnect themselves from the donor, and from the donation.

Thompson (2005) notes that an ontological choreography, including regulatory frameworks, maintains a connection between the woman and her body parts (i.e. eggs) when this becomes threatened and tenuous by physical separation. I argue that in the context of sperm donation, the anonymity regulation of sperm donors works in the opposite way. Not only does the law not ontologically choreograph a connection between the donor and his gametes when the two are disconnected, but it intentionally severs that link by black-boxing the production of sperm. Building on Thompson, I argue that the clinical regulatory, medical and institutional frameworks in place ontologically choreograph sperm donations in ways that disconnect the sperm from 
the donor. The couples who underwent medical treatment found that this assisted them in negotiating the intimate overtones of retrieving donor sperm.

\section{Discussion}

I have demonstrated in this paper that recipients of donor sperm experience that sperm donations give rise to dilemmas of intimacy and tensions related to sexual and intimate practices and relationships. Tensions have to be carefully managed in order for sperm donation not to spill over intimate and personal boundaries. The lesbian couples who self-arranged insemination intentionally set up arrangements with donors that created carefully structured routines organised in time and space. These routines can be understood to form a 'donation dance', a choreography that aims to accomplish the management of intimate, sexual and moral dilemmas. A process of separation and delineation produces a drawing out effect and a delay between the production of sperm and its retrieval. Through processes associated with medicalisation, institutionalisation and regulation, this drawing out effect is amplified in the clinic context, making it appear to be a 'cleaner' and less messy practice compared with self-arranged conception.

I argue that the choreography of sperm donation is rendered meaningful in complex and multilayered ways. The choreographies are ways for lesbian couples to manage the tension between having close contact with male sexuality and sperm (challenging their sexual boundaries and identities) and distancing themselves. They are also aimed at 'containing' the retrieval of donor sperm and managing the tensions and anxieties associated with crossing socially valued boundaries around privacy, masturbation, intimate bodies, sexual practices and interpersonal relationships. In that sense they are contextual (the anxieties are emphasised in non-clinical retrievals) and are likely to be fraught for any recipient of donor sperm, not only lesbian couples. The choreographies are, moreover, aimed at defining and defending the recipients' understandings of the interpersonal relationships at stake as well as maintaining the more general boundaries between private and public. They also represent a way of creating distance from the donor as a person with personal desires, a practice that links in with the tendency to render donor insemination, and sperm donors, invisible (Kirkman 2005).

The couples in my study tried to demarcate the donor from their process of conception as far as possible. They sought to make a sexual and intimate practice 
distant, impersonal and disengaged. The data seem to indicate that the couples were trying to negotiate needing a donor's sperm, but felt very reluctant to access it. How can we understand the character of the sperm donor and the sperm emerging as a result of the couples trying to resolve this dilemma? Thompson (2005) suggests that technology can both stand in partnership with, and in opposition to, personhood, and that a careful ontological choreography is required if women who undergo treatment with their own gametes are not to be objectified by the medical procedures applied in this process. Objectification is avoided by the maintenance of connections between the woman's body, her subjectivity and the trajectory of conception. I argue that the exact opposite process was made to happen in sperm donation where the linkages between the donor and the sperm were severed and made invisible. Following the line of thought of Thompson (2005) and Latour (1993), the donor became objectified through purification processes in place in the medical framework where the links between the donor, his subjectivity and the sperm were suspended. I argue that donation choreographies and the medical framework enabled couples to negotiate the subject-status of the donor, transforming the donor and the sperm from being personlike to being thing-like.

Finally it should be noted, however, that the process of purification and objectification of the donor and donor sperm is not and cannot be completed. Latour (1993) critiques the semblance of purified human and non-human relations and notes that there is also, simultaneously, an ongoing process of hybridisation. Although there are a multitude of processes of separation and delineation in the couples' accounts, the purification process is never entirely finalised. My study suggests that the anxieties associated with intimacy and donor sperm are perhaps lessened through the choreographies of donor sperm, and particularly so in clinics. However, drawing on Kirkman (2004), Burr (2009), and Grace, Daniels and Gillett (2008), donor insemination also raises social anxieties on a level of personal relationships and parental identities, and considerable ambivalence about donors lingers after conception and birth. Therefore, rather than understanding the choreographies as totally purifying and objectifying donor sperm, I argue that the practices of separating, delineating, delaying and drawing out enable the couples to manage, but never resolve, the tensions around sexuality, intimacy and personal life embedded in the process of retrieving sperm. 


\section{References}

Barney, S. (2005). Accessing medicalised donor sperm in the US and Britain: An historical narrative. Sexualities, 8(2), 205-220.

Becker, G. (2000). The Elusive Embryo. How Women and Men Approach New Reproductive Technologies. Berkeley: University of California Press.

Burr, J. (2009). Fear, fascination and the sperm donor as 'abjection' in interviews with heterosexual recipients of donor insemination. Sociology of Health \& Illness 31(5), 705-718.

Clarke, A. (1995). Modernity, postmodernity, and reproductive processes ca. 18901990 or 'Mommy, where do cyborgs come from anyway?'’. In C.H. Gray et al (Eds.), The Cyborg Handbook (pp. 139-155). New York: Routledge.

Clarke, A. et al (2003). Biomedicalisation: Technoscientific transformations of health, illness and U.S biomedicine. American Sociological Review, 68(2),161-194.

Daniels, K. and Haimes, E (1998) (Eds). Donor Insemination. International Social Science Perspectives. Cambridge: Cambridge University Press.

Donovan, C. (2006). Genetics, fathers and families: Exploring the implications of changing the law in favour of identifying sperm donors. Social and Legal Studies, 15(4), 494-510.

Donovan, C. and Wilson, A. (2008). Imagination and integrity: Decision-making among lesbian couples to use medically provided donor insemination. Culture, Health and Sexuality, 10(7), 649-665.

Douglas, M. (1966). Purity and Danger: An Analysis of Concepts of Pollution and Taboo. London: Routledge and Kegan Paul.

Edwards, J. (2000). Born and Bred: Idioms of Kinship and New Reproductive Technologies in England. Oxford: Oxford University Press. 
Emerson, J. (1970). Behavior in private places: Sustaining definitions of reality in gynecological examinations. Recent Sociology, 2, 74-97.

Franklin, S. (1997). Embodied Progress. A Cultural Account of Assisted Conception. London: Routledge.

Grace, V., Daniels, K. \& Gillett, W. (2008) 'The donor, the father, and the imaginary constitution of the family: Parents' constructions in the case of donor insemination', Social Science and Medicine, 66(2), 301-314.

Graham, H. (2007). Unequal Lives: Health and Socio-Economic Inequalities. Maidenhead: Open University Press.

Hargreaves, K. (2006). Constructing families and kinship through donor insemination. Sociology of Health \& Illness, 28(3), 261-283.

Human Fertilisation and Embryology Authority (2006). Licence Renewal Inspection Report for Treatment and Storage Centres James Cook. Available at <guide.hfea.gov.uk/guide/ShowPDF.aspx?ID=3447>. Accessed 9 December 2010.

Human Fertilisation and Embryology Authority (2009) Code of Practice $8^{\text {th }}$ Edition. Available at <http://www.hfea.gov.uk/code.html>. Accessed 9 December 2010.

Kirkman, M. (2004). Saviours and satyrs: ambivalence in narrative meanings of sperm provisions. Culture, Health and Sexuality, 6(4), 319-335.

Kirkman, M. (2005). Going home and forgetting about it. Donor insemination and the secrecy debate. In H.G. Jones \& M. Kirkman (Eds.), Sperm Wars (pp. 153-169). Sydney: ABC Books.

Latour, B. (1993). We Have Never Been Modern. New York: Harvester Wheatsheaf. Lieblich, A. et al. (1998). Narrative Research. Reading, Analysis and Interpretation. London: Sage.

Luce, J. (2010). Beyond Expectation. Lesbian/Bi/Queer Women and Assisted Conception. Toronto: University of Toronto Press. 
Mamo, L. (2007). Queering Reproduction. Achieving Pregnancy in the Age of Technoscience. Durham: Duke University Press.

Martin, E. (1991) 'The Egg and the Sperm: How Science Has Constructed a Romance Based on Stereotypical Male-Female Roles', Signs, 16(3), 485-501.

Mason, J. (1996). Qualitative Researching. London: Sage Publications.

Michelle, C. (2006). Transgressive technologies? Strategies of discursive containment in the representation and regulation of assisted reproductive technologies in Aotearoa/New Zealand'. Women's Studies International Forum 29(2), 109-124

Miles, M. \& Huberman, A. (1994). Qualitative Data Analysis. An Expanded Sourcebook. $2^{\text {nd }}$ ed. London: Sage Publications.

Morgan, D. (1996). Family Connections. An Introduction to Family Studies. Cambridge: Polity Press.

Murray, C., \& Golombok, S. (2003). To tell or not to tell: the decision-making process of egg-donation parents. Human Fertility, 6(2), 89-95.

Nordqvist, P. (2011). Dealing with sperm: comparing lesbians' clinical and nonclinical donor conception processes. Sociology of Health and Illness, 33(1): 114129.

Nordqvist, P. (under review). Origins and originators: lesbian couples negotiating parental identities and sperm donor conception. Culture, Health and Sexuality. (22 pages)

Ryan-Flood, R. (2009). Lesbian Motherhood. Gender, Families and Sexual Citizenship. Basingstoke: Palgrave Macmillan.

Strathern, M. (1992). Reproducing the Future. Essays on Anthropology, Kinship and the New Reproductive Technologies. Manchester: Manchester University Press. 
Thompson, C. (2005). Making Parents. The Ontological Choreography of Reproductive Technologies. Cambridge, Massachusetts: MIT.

Weeks, J. et al. (2001). Same Sex Intimacies: Families of Choice and Other Life Experiments. New York: Routledge. 